\title{
Mathematical model of generalized thermoelastic infinite medium with a spherical cavity and fractional order strain
}

\author{
Eman A. N. Al-Lehaibi
}

Mathematics Department, College of Science and Arts-Sharoura, Najran University, KSA.

\begin{abstract}
In this paper, a new mathematical model of a thermoelastic isotropic unbounded medium contains a spherical cavity thermally shocked under generalized thermo-elasticity with the fractional order strain model. The governing system of the partial differential equations has been derived in Laplace transform domain, and the inversion was done numerically by using the sum of Riemann approximation techniques. The numerical outputs of the displacement, the temperature, the stress, and the strain have been obtained and presented graphically. The fractional order parameter has an essential consequence on the stress, the strain, and the displacement distributions while its effect on the temperature increment distribution is very limited.
\end{abstract}

Keywords: Generalized thermo-elasticity, spherical cavity, fractional calculus, fractional strain.

2010 MSC: 74B20, 74F05, 35R11, 65R10.

(C)2019 All rights reserved.

\section{Nomenclature}

$\lambda, \mu$ : Lame's constants;

$\mathrm{C}_{\mathrm{E}}$ : specific heat at constant strain;

$\gamma=(3 \lambda+2 \mu) \alpha_{\mathrm{T}}$;

$\mathrm{T}$ : temperature;

$\theta=\left(T-T_{0}\right)$ temperature increment such that $\frac{|\theta|}{T_{0}}<<1$;

$e_{i j}$ : components of the strain tensor;

$\mathrm{K}$ : thermal conductivity;

$$
\begin{aligned}
& c_{o}=\sqrt{\frac{\lambda+2 \mu}{\rho}} ; \\
& \varepsilon=\frac{\gamma}{\rho C_{E}} ; \\
& \beta=\left(\frac{\lambda+2 \mu}{\mu}\right)^{1 / 2} .
\end{aligned}
$$

$\rho$ : density;

$\alpha_{\mathrm{T}}$ : the coefficient of linear thermal expansion;

$t$ : time;

$\mathrm{T}_{\mathrm{O}}$ : reference temperature;

$\sigma_{i j}$ : components of the stress tensor;

$u_{i}$ : components of the displacement vector;

$\tau_{0}:$ relaxation times;

$\eta=\frac{\rho C_{E}}{K}$

$\alpha=\frac{\gamma T_{o}}{\mu}$

Email address: dremanallehaibi@gmail.com (Eman A. N. Al-Lehaibi)

doi: $10.22436 /$ jnsa.012.01.03

Received: 2018-06-11 Revised: 2018-08-05 Accepted: 2018-08-31 


\section{Introduction}

The classical uncoupled model of thermo-elasticity offers two phenomena not agree with the physical observations. First, the equilibrium of heat conduction does not restrain any elastic terms. Second, the heat conduction equation generates infinite speeds of propagation for the thermal wave. Biot deduced the theory of coupled thermo-elasticity (CTE) which is coupled governing equations, and it eliminated the first paradox of the classical theory. Both theories still have the second paradox where the heat conduction equation for the coupled theory is parabolic type [2]. Lord and Shulman constructed a new law of heat conduction by replacing the classical Fourier's law [7]. Because of the heat conduction equation of this model is of the hyperbolic type, it predicts finite speeds of propagation for the thermal and the mechanical waves. The equations of motion and constitutive relations of this model are the same as those for the previous models.

Müller considered a new entropy inequality restriction on a class of constitutive equations [11]. New generalizations of this inequality were introduced by Green-Laws [5], Green-Lindsay [6], and Suhubi [14].

Erbay and Suhubi studied the longitudinal wave propagation in an infinite cylinder and got the equation of dispersion when the temperature of the cylinder surface was constant [3]. Problems of the generalized thermoelastic infinite cylindrical body with a hole were solved by Furukawa et al. [4] and Misra et al. $[9,10]$. Sherief and Anwar discussed a problem of two-dimensional generalized thermoelasticity problems for an infinitely long cylinder [13]. Youssef solved problems on generalized thermo-elasticity for an infinite material with a spherical cavity $[16,17]$. Al-Huniti et al. discussed the dynamical response of an elastic rod due to a moving heat source under the non-Fourier law of heat conduction [1].

Magin and Royston introduced the first model by using the fractional order derivative of the deformation of the material's behavior [8]. In this model, the zero-order of the derivative gives Hookean solid, and the one-order of the derivative gives Newtonian fluid while elastic and viscoelastic materials fill the intermediate range between zero and one [8]. The cartilage as an example is a sensitive type of tissue. It needs a multi-scale model that spans a wide range of collagen and proteoglycan molecules. The big challenge for the bioengineers is to improve the multi-scale tools that offer the macro-scale mechanical behavior of cartilage from micro-scale models [8].

Youssef constructed a new theory of thermo-elasticity based on fraction order of strain which is considered as a new modification to Duhamel-Neumann of stress-strain relation. Youssef solved the first mathematical model of thermo-elasticity with fractional order strain for a homogeneous isotropic onedimensional thermoelastic half-space based on different models of thermo-elasticity [18].

\section{The governing equations}

We assume a perfectly conducting thermoelastic infinite body with a spherical cavity occupies the region $R \leqslant r<\infty$ of an isotropic homogeneous medium whose state can be expressed in terms of the space variable $r$ and the time variable $t$ such that all of the field functions vanish at infinity. A spherical system of coordinates $(r, \varphi, \psi)$ will be used, and due to the symmetric construction, the problem is onedimensional. It is assumed that there are no external body that forces or heat suppliers in the medium and it is initially quiescent.

Thus the field equations in spherical one-dimensional take the form [18]:

$$
(\lambda+2 \mu)\left(1+\tau^{\xi} D_{t}^{\xi}\right) \frac{\partial e}{\partial r}-\gamma \frac{\partial \theta}{\partial r}=\rho \frac{\partial^{2} u}{\partial t^{2}}
$$

and

$$
\nabla^{2} \theta=\frac{\rho C_{E}}{K}\left(\frac{\partial}{\partial t}+\tau_{0} \frac{\partial^{2}}{\partial t^{2}}\right) \theta+\frac{T_{0} \gamma}{K}\left(\frac{\partial}{\partial t}+\tau_{0} \frac{\partial^{2}}{\partial t^{2}}\right)\left(1+\tau^{\xi} D_{t}^{\xi}\right) e,
$$

where

$$
\nabla^{2}=\frac{1}{r^{2}} \frac{\partial}{\partial r}\left(r^{2} \frac{\partial}{\partial r}\right)
$$


The constitutive equations take the following forms [18]:

$$
\begin{aligned}
\sigma_{r r} & =2 \mu\left(1+\tau^{\xi} D_{t}^{\xi}\right) \frac{\partial u}{\partial r}+\lambda\left(1+\tau^{\xi} D_{t}^{\xi}\right) e-\gamma \theta, \\
\sigma_{\psi \psi} & =\sigma_{\varphi \varphi}=2 \mu\left(1+\tau^{\xi} D_{t}^{\xi}\right) \frac{u}{r}+\lambda\left(1+\tau^{\xi} D_{t}^{\xi}\right) e-\gamma \theta, \\
\sigma_{r \varphi} & =\sigma_{r \psi}=\sigma_{\varphi \psi}=0, \\
e & =\frac{1}{r^{2}} \frac{\partial\left(r^{2} u\right)}{\partial r} .
\end{aligned}
$$

The fractional derivative with respect to the time $D_{t}^{\xi}$ is defined as $[8,18]$ :

$$
D_{t}^{\xi} f(t)=\frac{\partial^{\xi} f(t)}{\partial t^{\xi}}=\frac{1}{\Gamma(1-\xi)} \int_{0}^{t} \frac{f^{\prime}(x)}{(t-\chi)^{\xi}} d x, \quad 0 \leqslant \xi<1 .
$$

Applying the following non-dimensional variables [18]:

$$
r^{\prime}=c_{o} \eta r, u^{\prime}=c_{o} \eta u, t^{\prime}=c_{o}^{2} \eta t, \tau_{0}^{\prime}=c_{o}^{2} \eta \tau_{0}, \tau^{\prime}=c_{o}^{2} \eta \tau R^{\prime}=c_{o} \eta R, \theta^{\prime}=\frac{T-T_{o}}{T_{o}}, \sigma^{\prime}=\frac{\sigma}{\mu},
$$

where $c_{o}^{2}=\frac{\lambda+2 \mu}{\rho}$ and $\eta=\frac{\rho C_{E}}{K}$.

Equations (2.1), (2.2), (2.3), and (2.4) take the following forms (where the primes are suppressed for simplicity):

$$
\begin{gathered}
\left(1+\tau^{\xi} D_{t}^{\xi}\right) \frac{\partial e}{\partial r}-b \frac{\partial \theta}{\partial r}=\frac{\partial^{2} u}{\partial t^{2}} \\
\nabla^{2}\left(1+\tau^{\xi} D_{t}^{\xi}\right) e-b \nabla^{2} \theta=\frac{\partial^{2} e}{\partial t^{2}} \\
\nabla^{2} \theta=\left(\frac{\partial}{\partial t}+\tau_{0} \frac{\partial^{2}}{\partial t^{2}}\right) \theta+\varepsilon\left(\frac{\partial}{\partial t}+\tau_{0} \frac{\partial^{2}}{\partial t^{2}}\right)\left(1+\tau^{\xi} D_{t}^{\xi}\right) e, \\
\sigma_{r r}=\beta^{2}\left(1+\tau^{\xi} D_{t}^{\xi}\right) e-4\left(1+\tau^{\xi} D_{t}^{\xi}\right) \frac{u}{r}-\alpha \theta, \\
\sigma_{\psi \psi}=\sigma_{\varphi \varphi}=\left(\beta^{2}-2\right)\left(1+\tau^{\xi} D_{t}^{\xi}\right) e+2\left(1+\tau^{\xi} D_{t}^{\xi}\right) \frac{u}{r}-\alpha \theta,
\end{gathered}
$$

where $\gamma=(3 \lambda+2 \mu) \alpha_{\mathrm{T}}, \alpha=\frac{\gamma \mathrm{T}_{\mathrm{o}}}{\mu}, \varepsilon=\frac{\gamma}{\rho \mathrm{C}_{\mathrm{E}}}, \beta=\left(\frac{\lambda+2 \mu}{\mu}\right)^{1 / 2}$, and $\mathrm{b}=\frac{\alpha}{\beta^{2}}$.

We use the Laplace transform of both sides of the last equations which is defined in the form

$$
\bar{f}(s)=\int_{0}^{\infty} f(t) e^{-s t} d t
$$

Hence, we obtain

$$
\begin{aligned}
\nabla^{2}\left(1+\tau^{\xi} s^{\xi}\right) \bar{e} & =s^{2} \bar{e}+b \nabla^{2} \bar{\theta} \\
\nabla^{2} \bar{\theta} & =s\left(1+\tau_{0} s\right) \bar{\theta}+\varepsilon s\left(1+\tau_{0} s\right)\left(1+\tau^{\xi} s^{\xi}\right) \bar{e} \\
\bar{\sigma}_{r r} & =\beta^{2}\left(1+\tau^{\xi} s^{\xi}\right) \bar{e}-4\left(1+\tau^{\xi} s^{\xi}\right) \frac{\bar{u}}{r}-\alpha \bar{\theta} \\
\bar{\sigma}_{\psi \psi} & =\bar{\sigma}_{\varphi \varphi}=\left(\beta^{2}-2\right)\left(1+\tau^{\xi} s^{\xi}\right) \bar{e}+2\left(1+\tau^{\xi} s^{\xi}\right) \frac{\bar{u}}{r}-\alpha \bar{\theta},
\end{aligned}
$$

and

$$
\bar{e}=\frac{1}{r^{2}} \frac{\partial\left(r^{2} \bar{u}\right)}{\partial r}
$$


The rule for the Laplace transforms of the Riemann-Liouville fractional derivative, reads from [12] as follows:

$$
L\left\{D_{t}^{\xi} f(t)\right\}=s^{\xi} L\{f(t)\}=s^{\xi} \bar{f}(s), \quad \xi>0,
$$

where all the initial state functions are zero.

By rewriting equations (2.5) and (2.6), we get

$$
\left[\nabla^{2}-\alpha_{1}\right] \bar{e}=\alpha_{2} \nabla^{2} \bar{\theta}
$$

and

$$
\left[\nabla^{2}-\alpha_{3}\right] \bar{\theta}=\alpha_{4} \bar{e}
$$

where $\alpha_{1}=\frac{\mathrm{s}^{2}}{\left(1+\tau^{\xi} s^{\xi}\right)}, \alpha_{2}=\frac{\mathrm{b}}{\left(1+\tau^{\xi} s^{\xi}\right)}, \alpha_{3}=\left(s+\tau_{0} s^{2}\right)$, and $\alpha_{4}=\varepsilon\left(s+\tau_{0} s^{2}\right)\left(1+\tau^{\xi} s^{\xi}\right)$.

Eliminating $\bar{e}$ from equations (2.9) and (2.10), we obtain

$$
\left[\nabla^{4}-\mathrm{L} \nabla^{2}+\mathrm{M}\right] \bar{\theta}=0
$$

where

$$
\mathrm{L}=\alpha_{1}+\alpha_{3}-\alpha_{2} \alpha_{4}, M=\alpha_{1} \alpha_{3} .
$$

Similarly, we can get the following equation

$$
\left[\nabla^{4}-\mathrm{L} \nabla^{2}+\mathrm{M}\right] \overline{\mathrm{e}}=0
$$

The solutions of equations (2.11) and (2.12) which are bounded at infinity, can be written in the form

$$
\bar{\theta}=\sum_{i=1}^{2} A_{i}\left(p_{i}^{2}-\alpha_{1}\right) \frac{e^{-p_{i} r}}{r},
$$

and

$$
\bar{e}=\sum_{i=1}^{2} B_{i} \frac{e^{-p_{i} r}}{r},
$$

where $A_{1}, A_{2}, B_{1}$, and $B_{2}$ all are parameters depending on the $s$ of the Laplace transform and $p_{1}^{2}$ and $p_{2}^{2}$ are the roots of the characteristic equation

$$
\mathrm{p}^{4}-\mathrm{Lp} \mathrm{p}^{2}+\mathrm{M}=0
$$

Using equation (2.9), we obtain

$$
B_{i}=\alpha_{2} p_{i}^{2} A_{i}, i=1,2
$$

Hence, we have

$$
\bar{e}=\alpha_{2} \sum_{i=1}^{2} p_{i}^{2} A_{i} \frac{e^{-p_{i} r}}{r} .
$$

Substituting from equation (2.14) into equation (2.8), we obtain

$$
\bar{u}=\alpha_{2} \sum_{i=1}^{2} A_{i}\left(1+r p_{i}\right) \frac{e^{-p_{i} r}}{r^{2}} .
$$

Substituting from equations (2.13), (2.14), and (2.15) into equation (2.7), we obtain

$$
\bar{\sigma}_{\mathrm{rr}}=\sum_{i=1}^{2} \mathrm{~L}_{\mathrm{i}}(\mathrm{r}) \mathrm{A}_{\mathrm{i}} \mathrm{e}^{-\mathrm{p}_{\mathrm{i}} \mathrm{r}},
$$


where $L_{i}(r)=\frac{\alpha\left(\alpha_{1}-p_{i}^{2}\right)}{r}+\alpha_{2}\left(1+\tau^{\xi} s^{\xi}\right)\left(\frac{p_{i}^{2} \beta^{2}}{r}+\frac{4\left(p_{i} r+1\right)}{r^{3}}\right), i=1,2$.

To get the complete solutions in the Laplace transform domain, we will assume the bounding plane of the cavity $(r=R)$ traction free and subjected to a thermal shock as follows:

$$
\theta(R, t)=\theta_{0} H(t),
$$

where $\theta_{0}$ is constant and $\mathrm{H}(\mathrm{t})$ is the well-known Heaviside function (unite step function).

After using Laplace transform, we have

$$
\bar{\theta}(R, s)=\frac{\theta_{0}}{s} .
$$

Also, we have

$$
\sigma(R, t)=0
$$

After using Laplace transform, we have

$$
\bar{\sigma}(R, s)=0 .
$$

Applying the last two conditions (2.16) and (2.17), we obtain

$$
A_{1}=\frac{L_{22} R \theta_{0} e^{p_{1} R}}{s\left[L_{11}\left(\alpha_{1}-p_{2}^{2}\right)-L_{22}\left(\alpha_{1}-p_{1}^{2}\right)\right]},
$$

and

$$
A_{2}=\frac{-L_{11} R \theta_{0} e^{p_{2} R}}{s\left[L_{11}\left(\alpha_{1}-p_{2}^{2}\right)-L_{22}\left(\alpha_{1}-p_{1}^{2}\right)\right]}
$$

where

$$
\begin{aligned}
& \mathrm{L}_{11}=\frac{\alpha\left(\alpha_{1}-p_{1}^{2}\right)}{\mathrm{R}}+\alpha_{2}\left(1+\tau^{\xi} s^{\xi}\right)\left(\frac{\beta^{2}}{\mathrm{R}}+\frac{4\left(p_{1} \mathrm{R}+1\right)}{p_{1}^{2} \mathrm{R}^{3}}\right), \\
& \mathrm{L}_{22}=\frac{\alpha\left(\alpha_{1}-p_{2}^{2}\right)}{\mathrm{R}}+\alpha_{2}\left(1+\tau^{\xi} s^{\xi}\right)\left(\frac{\beta^{2}}{\mathrm{R}}+\frac{4\left(p_{2} \mathrm{R}+1\right)}{p_{2}^{2} \mathrm{R}^{3}}\right),
\end{aligned}
$$

which complete the solution of the problem in the Laplace transform domain.

\section{Numerical inversion of the Laplace transform}

To obtain the conductive temperature increment, the dynamical temperature increment, the displacement, and the stress distributions in the time domain, the Riemann-sum approximation techniques will be used where any function in Laplace transform domain can be inverted to the time domain as [15]:

$$
f(t)=\frac{e^{\kappa t}}{t}\left[\frac{1}{2} \bar{f}(\kappa)+\operatorname{Re} \sum_{n=1}^{N}(-1)^{n} \bar{f}\left(\kappa+\frac{i n \pi}{t}\right)\right],
$$

where Re is the real part and $i$ is the imaginary number unit. For faster convergence, numerous numerical experiments have shown that the value of $\kappa$ satisfies the relation $\kappa t \approx 4.7$, [15].

\section{Numerical results and discussion}

Assume a numerical example for which computational results are given. For this purpose, copper is taken as the thermoelastic material for which we take the following values of the different physical constants [16-18]:

$$
\begin{aligned}
& \mathrm{K}=386 \mathrm{~kg} \mathrm{~m} \mathrm{k}^{-1} \mathrm{~s}^{-3}, \quad \alpha_{\mathrm{T}}=1.78(10)^{-5} \mathrm{k}^{-1}, \quad \rho=8954 \mathrm{~kg} \mathrm{~m}^{-3}, \mathrm{~T}_{\mathrm{o}}=293 \mathrm{k}, \\
& \mathrm{C}_{\mathrm{E}}=383.1 \mathrm{~m}^{2} \mathrm{k}^{-1} \mathrm{~s}^{-2}, \quad \mu=3.86(10)^{10} \mathrm{~kg} \mathrm{~m}^{-1} \mathrm{~s}^{-2}, \quad \lambda=7.76(10)^{10} \mathrm{~kg} \mathrm{~m}^{-1} \mathrm{~s}^{-2} \text {. }
\end{aligned}
$$


From the above values, we get the non-dimensional values of the problem as:

$$
\mathrm{b}=0.010471, \quad \alpha=0.0418853, \quad \varepsilon=1.6086, \quad \beta^{2}=4, \quad \mathrm{R}=1.0, \quad \tau_{0}=0.02, \quad \mathrm{t}=0.3 .
$$

The numerical results have been represented in figures with a wide range of the radial distance $r$ starting from the cavity $r=R=1.0$ up to $r=2.0$ and with different values of a parameter of the fractional order of the strain $\xi=0.0,0.5,1.0$ including the case of with/without fractional order parameter $\tau=$ $0.0,0.01$.

Figure 1 shows that the fractional order parameter of the strain has a tiny effect on the temperature distribution where the difference between the values of the temperature in the context of fractional order strain and the context of usual strain is of order $10^{-6}$. So the curves of all the cases that we mentioned them are almost identical.

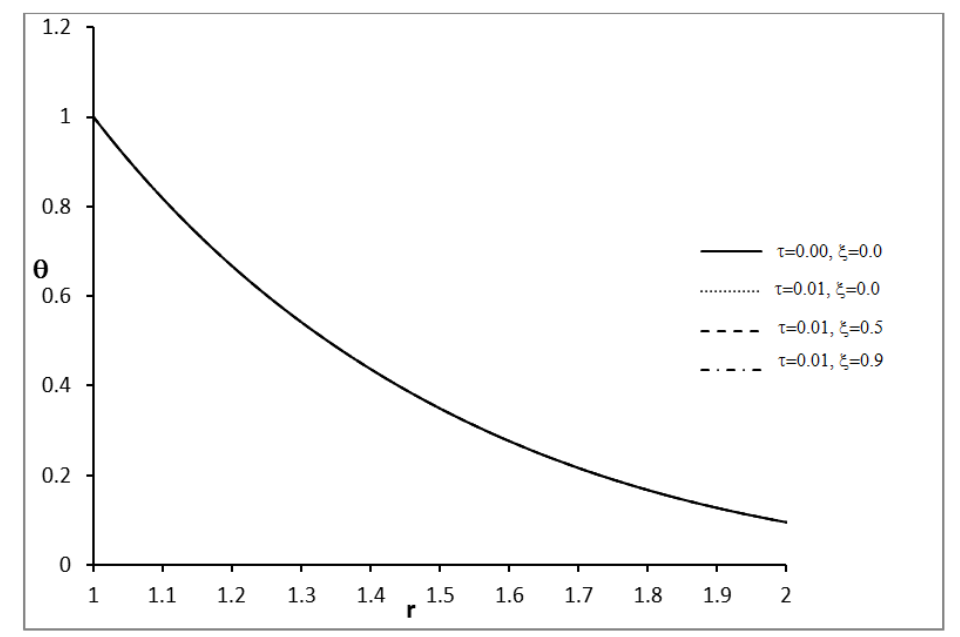

Figure 1: The temperature distribution with different values of fractional order parameter.

Figures 2, 3, and 4 show that the strain, the displacement, and the stress distributions respectively, and we can note that the fractional strain order parameter has significant effects. The absolute value of the peak point of the strain, the displacement, and the stress increases when the value of the fractional order parameter increases. We can note that also the difference between the cases of the usual strain and the fractional strain is apparent.

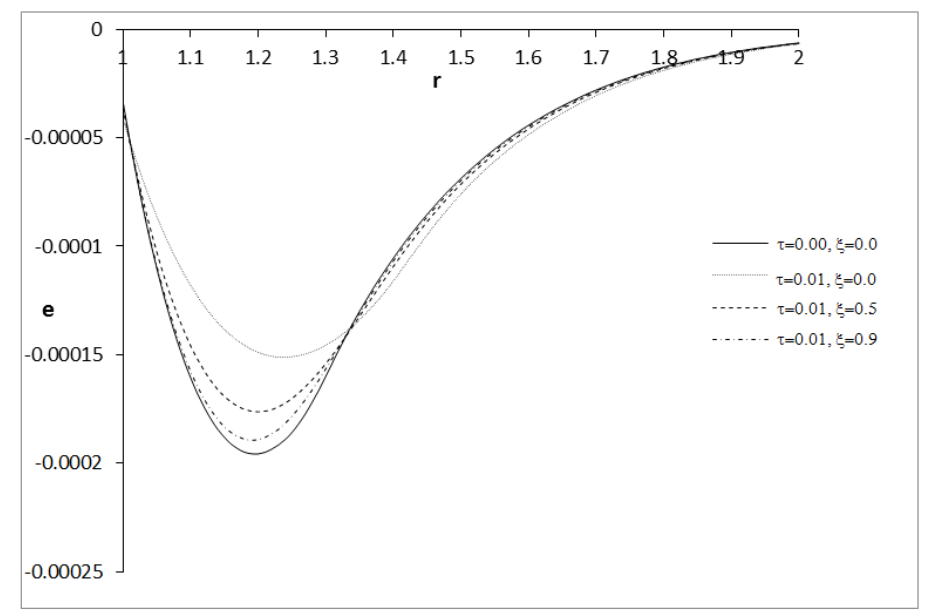

Figure 2: The strain distribution with different values of fractional order parameter. 


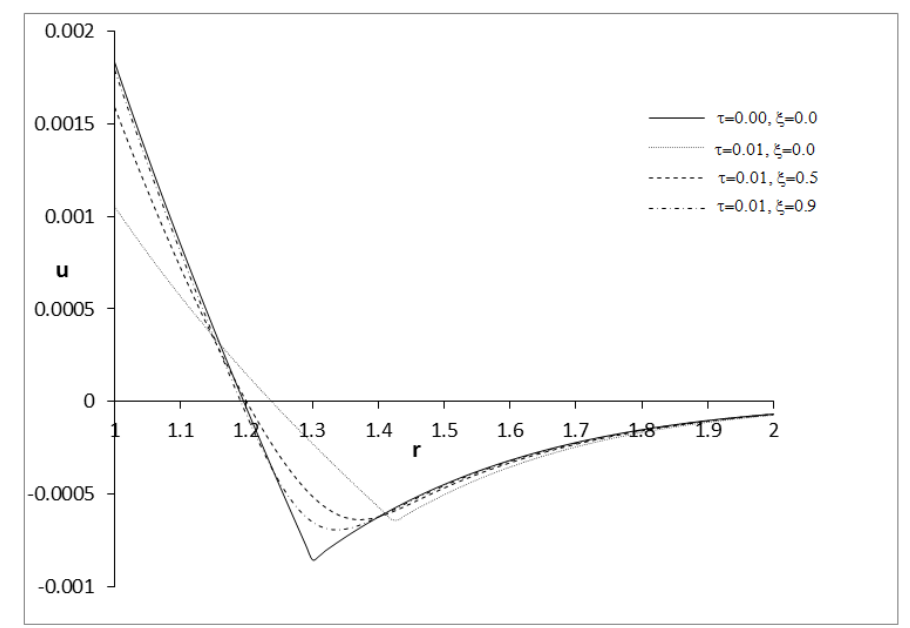

Figure 3: The displacement distribution with different values of fractional order parameter.

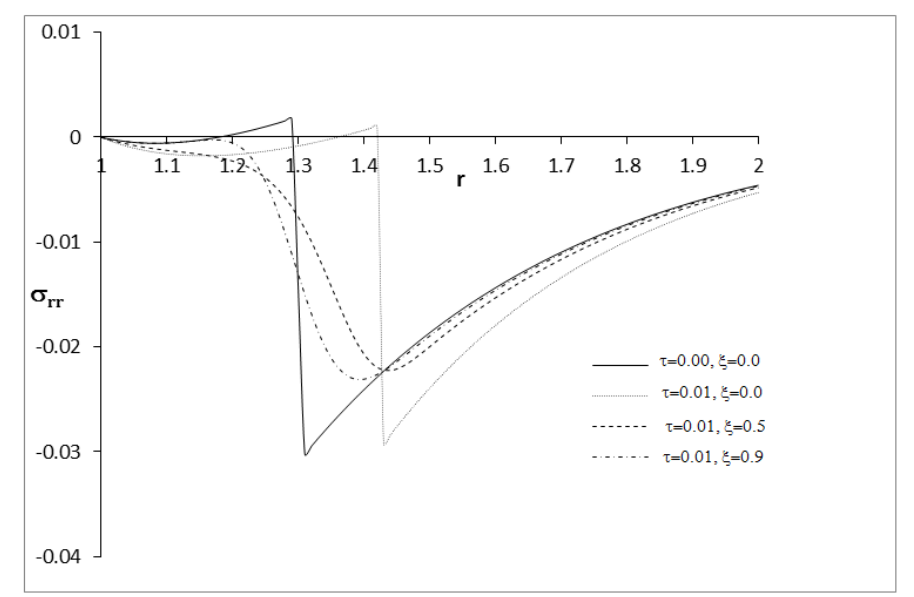

Figure 4: The radial stress distribution with different values of fractional order parameter.

\section{Conclusions}

The theory of generalized thermo-elasticity with fractional order strain introduces new results for thermoelastic materials when the stress-strain relation changes from a linear stage to the damper stage $0 \leqslant \xi<1.0$.

The fractional order parameter has significant effects on all studied mechanical fields and tiny effect on the temperature.

\section{Acknowledgment}

The authors are grateful for the supports and introduce a fund for this work provided by Deanship of Scientific Research at the Najran University, KSA with grant number NU/ESCI/15/071. This funding did not lead to any conflict of interests regarding the publication of this manuscript.

\section{References}

[1] N. S. Al-Huniti, M. Al-Nimr, M. Naji, Dynamic response of a rod due to a moving heat source under the hyperbolic heat conduction model, J. Sound Vib., 242 (2001), 629-640. 1

[2] M. A. Biot, Thermoelasticity and irreversible thermodynamics, J. Appl. Phys., 27 (1956), 240-253. 1 
[3] S. Erbay, E. S. Şuhubi, Longitudinal wave propagation in a generalized thermoelastic cylinder, J. Therm. Stresses, 9 (1986), 279-295. 1

[4] T. Furukawa, N. Noda, F. Ashida, Generalized thermoelasticity for an infinite body with a circular cylindrical hole, JSME Int. J. I-Solid M., 33 (1990), 26-32. 1

[5] A. E. Green, N. Laws, On the entropy production inequality, Arch. Rational Mech. Anal., 45 (1972), 47-53. 1

[6] A. E. Green, K. A. Lindsay, Thermoelasticity, J. Elasticity, 2 (1972), 1-7. 1

[7] H. W. Lord, Y. Shulman, A generalized dynamical theory of thermoelasticity, J. Mech. Phys. Solids, 15 (1967), $299-309$. 1

[8] R. L. Magin, T. J. Royston, Fractional-order elastic models of cartilage: A multi-scale approach, Commun. Nonlinear Sci. Numer. Simul., 15 (2010), 657-664. 1, 2

[9] J. C. Misra, N. C. Chattopadhyay, S. C. Samanta, Thermoviscoelastic waves in an infinite aeolotropic body with a cylindrical cavity-a study under the review of generalised theory of thermoelasticity, Computers \& structures, 52 (1994), 705-717. 1

[10] J. C. Misra, S. C. Samanta, A. K. Chakrabarti, S. C. Misra, Magnetothermoelastic interaction in an infinite elastic continuum with a cylindrical hole subjected to ramp-type heating, Int. J. Eng. Sci., 29 (1991), 1505-1514. 1

[11] I. Mller, The Coldness, a Universal Function in Thermo-Elastic Solids, Arch. Rat. Mech. Anal., 41 (1971), $319-332.1$

[12] J. L. Schiff, The Laplace transform: theory and applications, Springer Science \& Business Media, New York, (2013). 2

[13] H. H. Sherief, M. N. Anwar, Two-dimensional generalized thermoelasticity problem for an infinitely long cylinder, J. Thermal Stresses, 17 (1994), 213-227. 1

[14] E. S. Şuhubi, Thermoelastic solids: Continuum Mechanics of Single-Substance Bodies, Elsevier, 1975 (1975), $173-265.1$

[15] D. Y. Tzou, Macro-to microscale heat transfer: the lagging behavior, John Wiley \& Sons, U.S.A., (2014). 3, 3

[16] H. M. Youssef, Dependence of modulus of elasticity and thermal conductivity on reference temperature in generalized thermoelasticity for an infinite material with a spherical cavity, Appl. Math. Mech., 26 (2005), 470-475. 1, 4

[17] H. M. Youssef, State-space approach on generalized thermoelasticity for an infinite material with a spherical cavity and variable thermal conductivity subjected to ramp-type heating, Can. Appl. Math. Q., 13 (2005), 369-390. 1

[18] H. M. Youssef, Theory of generalized thermoelasticity with fractional order strain, J. Vib. Control, 22 (2016), $3840-3857$. $1,2,2,2,2,4$ 